\title{
Allicin attenuates lipopolysaccharide-induced acute lung injury in neonatal rats via the PI3K/Akt pathway
}

\author{
XUDONG WANG, CHAO ZHANG, CHAO CHEN, YI GUO, XIAOYAN MENG and CHEN KAN \\ Department of Critical Care Medicine, The Second Affiliated Hospital of Xuzhou Medical University, \\ Xuzhou, Jiangsu 221006, P.R. China
}

Received April 10, 2017; Accepted December 1, 2017

DOI: $10.3892 / \mathrm{mmr} .2018 .8693$

\begin{abstract}
Allicin is an oxygenated carotenoid derivative that exhibits strong antioxidant activity, which effectively removes reactive oxygen species from the body and has important roles in disease prevention and treatment. Therefore, the present study aimed to investigate whether allicin attenuates lipopolysaccharide (LPS)-induced acute lung injury (ALI) in neonatal rats and the potential underlying mechanisms. An LPS-induced ALI neonatal rat model was utilized to assess the therapeutic value and mechanisms of allicin. Following allicin treatment, increases in lung wet/dry ratio and the lung protein concentration were significantly suppressed in LPS-induced ALI neonatal rats. Furthermore, ELISA results demonstrated that allicin significantly reduced the levels of malondialdehyde, tumor necrosis factor- $\alpha$ and interleukin-6, and increased superoxide dismutase activity, in the bronchoalveolar lavage fluid of LPS-treated rats. Additionally, allicin administration increased the protein expression of Bcl-2 and reduced the activity of caspase-3/-9, as determined by western blotting or ELISA, respectively, and increased phosphatidylinositol 3-kinase (PI3K) and phosphorylated-Akt protein levels, in LPS-treated ALI neonatal rats. The results of the present study indicate that allicin attenuate LPS-induced ALI in neonatal rats by ameliorating oxidative stress, inflammation and apoptosis via the PI3K/Akt pathway. Allicin may be used for development of a novel drug for treatment of ALI.
\end{abstract}

\section{Introduction}

Comprehensive studies on acute respiratory distress syndrome (ARDS) have revealed that ARDS is the most severe stage of the continuous pathological process of acute lung injury

Correspondence to: Dr Xudong Wang, Department of Critical Care Medicine, The Second Affiliated Hospital of Xuzhou Medical University, 32 Meijian Road, Xuzhou, Jiangsu 221006, P.R. China E-mail: zwygavvbwn@163.com

Key words: allicin, acute lung injury, oxidative stress, inflammation, apoptosis, phosphatidylinositol 3-kinase, Akt
(ALI); ALI is observed in all cases of ARDS, however ALI does necessarily develop into ARDS. It is also established that ALI is implicated in system inflammatory response syndrome (SIRS) $(1,2)$. The lung is vulnerable to damage and a role in SIRS (3). ALI commonly leads to severe hypoxemia, thereby leading to the dysfunction of other organs and potentially multiple organ failure (4). Therefore, ALI is the initial stage in the whole pathological process of SIRS, which results in organ dysfunction and subsequent multiple organ failure.

Newborns, particularly premature children, are prone to ALI, which may develop into ARDS. In severe cases, bronchopulmonary dysplasia impairs lung development, which is closely associated with the development of lung maturity (5). ALI may initially develop in utero and continue following birth. Pulmonary inflammation can cause lung damage and lead to ALI (5). The lung of a newborn is in cyst and alveolar period, which is immature (6). Pulmonary surfactant is observed after 28 weeks gestation, meaning that premature children are vulnerable to ALI and the further development and maturation are affected. A lower gestational age is associated with an increased risk of lung damage $(7,8)$.

Allicin is a type of volatile, oily matter that is extracted from spherical garlic bulbs and is the major component of garlic biological activity. Allicin is reported to exhibit various biological functions and physiological effects, including anti-inflammation, anti-cancer, cholesterol-lowering, anti-platelet aggregation and liver-protection effects, and also functions in preventing heart and vascular diseases and lowering blood pressure (9-14). The aim of the current study was to investigate whether allicin attenuates lipopolysaccharide (LPS)-induced ALI in neonatal rats and whether these beneficial actions may be mediated by ameliorating oxidative stress, inflammation and apoptosis.

\section{Materials and methods}

Animals and ALI model. Male Sprague-Dawley neonatal rats (weight, 5-30 g, 1 week old) were provided by the Experimental Animal Center of Xuzhou Medical University (Xuzhou, China) and kept at a temperature of $23 \pm 1^{\circ} \mathrm{C}$, $55-60 \%$ humidity, $0.038 \% \mathrm{CO}_{2}$, on a $12 \mathrm{~h} \mathrm{light/dark} \mathrm{cycle}$ with food and water available ad libitum. The present study was approved by the Institutional Animal Care and Use Committee of The Second Affiliated Hospital of Xuzhou 
Medical University. A total of 60 Sprague-Dawley rats were randomly divided into three groups as follows: Sham group $(n=20)$, model group $(n=20)$ and model + allicin group $(n=20)$. All rat was anesthetizated using $5 \mathrm{mg} / \mathrm{kg}$ pentobarbital tail intravenous injection. In the sham groups, rats only received normal saline.

ALI model rats received $5 \mathrm{mg} / \mathrm{kg}$ LPS (Sigma-Aldrich; Merck KGaA, Darmstadt, Germany). In the model+allicin treatment group, rats received $80 \mathrm{mg} / \mathrm{kg}$ allicin (Sigma-Aldrich; Merck KGaA) via tail intravenous injection for 8 days (15) after the ALI model was established. Following induction of the ALI model and confirmation using histological examination, the number of rats in each group was recorded as NO of LPS at 6, 12, 18, 24, 30, 36, 42 and $48 \mathrm{~h}$. Mortality rate was calculated as follows: [(total number - NO of LPS)/total number] x 100, where total number refers to the number of rats in each group at the start of the experiment $(\mathrm{n}=20)$. The chemical structure of allicin is presented in Fig. 1.

Lung wet/dry (W/D) ratio of LPS-treated rats and the lung concentration of proteins. The rats were euthanized using intravenous injection of $35 \mathrm{mg} / \mathrm{kg}$ pentobarbital and sacrificed using decollation. The thorax was opened and the whole lungs were immediately removed. The superior lobe of the right lung was harvested, weighed and dried at $80^{\circ} \mathrm{C}$ for $48 \mathrm{~h}$, and subsequently reweighed to calculate the W/D weight ratio of the lung tissue. Bronchoalveolar lavage fluid (BALF) was collected to determine the lung concentration of proteins via the BCA method (Wuhan Boster Biological Technology, Co., Ltd., Wuhan, China.)

Histological examination. Histological examination was used to determine whether the induction of the model was successful. The rats were euthanized using intravenous injection of $35 \mathrm{mg} / \mathrm{kg}$ pentobarbital and decapitation. Right lung tissue was immersed in $10 \%$ neutral phosphate-buffered formaldehyde fixative for $24 \mathrm{~h}$ at room temperature and embedded in paraffin. Tissue was cut into $4 \mu \mathrm{m}$ sections and stained with hematoxylin and eosin (HE) for $15 \mathrm{~min}$ at room temperature. Tissue sections were subsequently observed using a Nikon SMZ 1500 light microscope (magnification, x40; Nikon Corporation, Tokyo, Japan).

Oxidative stress and inflammation. The left lung of each rat was harvested and washed with ice-cold PBS. The volume of BALF was similar in each group and centrifuged at $1,200 \mathrm{x} \mathrm{g}$ for $10 \mathrm{~min}$ at $4^{\circ} \mathrm{C}$. The supernatant was collected to determine the levels of interferon (IFN)- $\gamma$, caspase-3 activity, caspase-9 activity, glutathione (GSH), glutathione peroxidase (GSH-PX), malondialdehyde (MDA), tumor necrosis factor (TNF)- $\alpha$ and interleukin (IL)-6, $-1 \beta$ and -10 levels, and superoxide dismutase (SOD) activity, using rat IFN- $\gamma$ (cat. no. H025), caspase-3 activity (cat. no. G015), caspase-9 activity (cat. no. G018), GSH (cat. no. A006-2), GSH-PX (cat. no. A005), MDA (cat. no. A003-1), TNF- $\alpha$ (cat. no. H052), IL-1 $\beta$ (cat. no. H002), IL-10 (cat. no. H009), IL-6 (cat. no. H007) and SOD (cat. no. A001-1-1) ELISA kits (Wuhan Boster Biological Technology, Co., Ltd.). The concentration of proteins was measured using the BCA method.

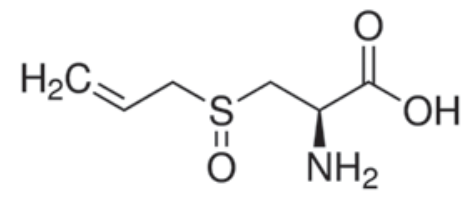

Figure 1. The chemical structure of allicin.

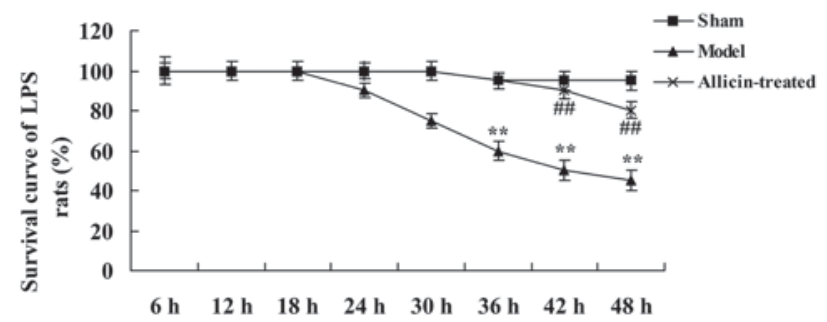

Figure 2. Effect of allicin on the survival rate of acute lung injury model neonatal rats. Data are presented as the mean \pm standard deviation $(n=3)$. ${ }^{* *} \mathrm{P}<0.01$ vs. sham group, ${ }^{\# \#} \mathrm{P}<0.01$ vs. model group. LPS, lipopolysaccharide.

Western blotting. BALF was collected following centrifugation at $10,000 \times \mathrm{g}$ for $10 \mathrm{~min}$ at $4^{\circ} \mathrm{C}$ to determine the concentration of proteins via the BCA method. An equal amount of protein $(50 \mu \mathrm{g})$ was separated by $10-12 \%$ SDS-PAGE, and transferred to and immobilized on a nitrocellulose membrane (Bio-Rad Laboratories, Inc., Hercules, CA, USA). The nitrocellulose membrane was blocked by incubation with PBS containing 5\% non-fat dried milk for $2 \mathrm{~h}$ at room temperature and subsequently incubated with primary antibodies against COX-2 (cat. no. 12282; 1:2,000; Cell Signaling Technology, Inc), NF-кB (cat. no. 8242; 1:2,000; Cell Signaling Technology, Inc), PI3K (cat. no. 4249; 1:2,000; Cell Signaling Technology, Inc), Akt (cat. no. 4691; 1:2,000; Cell Signaling Technology, Inc), p-Akt (cat. no. 4060; 1:2,000; Cell Signaling Technology, Inc), GAPDH (cat. no. D110016; 1:5,000; Sangon Biotech Co., Ltd., Shanghai, China) overnight at $4^{\circ} \mathrm{C}$. The nitrocellulose membrane was washed three times with TBS containing $0.1 \%$ Tween- 20 and incubated with horseradish peroxidase-conjugated anti-rabbit or anti-mouse secondary antibody (cat. nos. sc-2004 and sc-2005 respectively, both 1:5,000; Santa Cruz Biotechnology, Inc., Dallas, TX, USA) for $2 \mathrm{~h}$ at room temperature. Subsequently, the membrane was incubated with chemiluminescence reagent (ECL Plus Western Blotting Detection System; GE Healthcare, Chicago, IL, USA). Proteins were quantitatively analyzed using Image-Pro Plus 6.0 software (Media Cybernetics, Inc., Rockville, MD, USA).

Statistical analysis. Data are presented as the mean \pm standard deviation and were analyzed using SPSS 19 (IBM Corp., Armonk, NY, USA). All data were analyzed by one-way analysis of variance followed by Dunnett's post hoc test. $\mathrm{P}<0.05$ was considered to indicate a statistically significant difference.

\section{Results}

Effect of allicin on the mortality rate of LPS-treated neonatal rats. The survival of rats in the ALI model group began to 

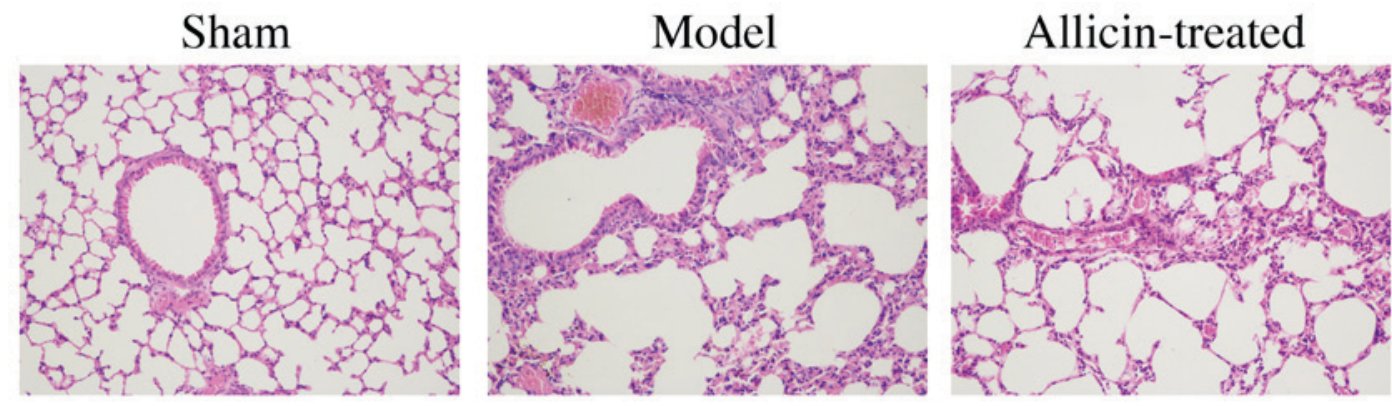

Figure 3. Effect of allicin on the histological examination of acute lung injury model neonatal rats (magnification, $\mathrm{x} 40$ ).

A

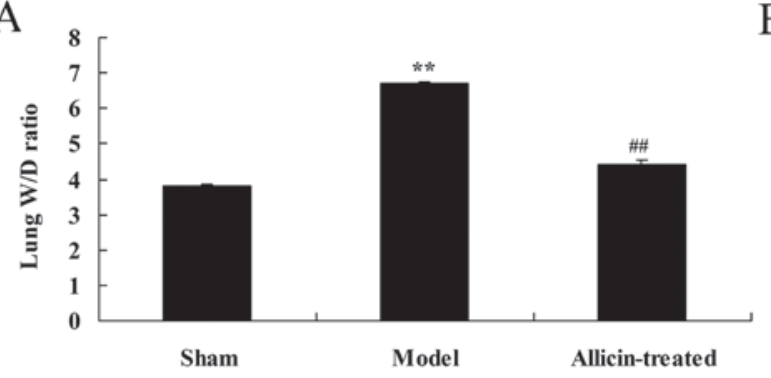

$\mathrm{B}$

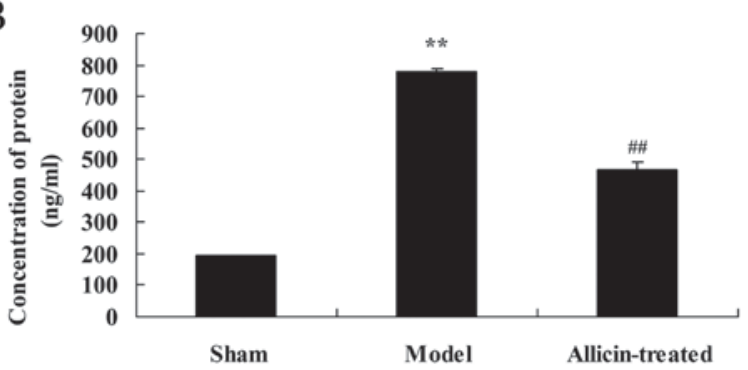

Figure 4. Effect of allicin on lung W/D ratio and protein concentration in ALI neonatal rats. Allicin significantly reduced (A) lung W/D ratio and (B) lung protein concentration in ALI model neonatal rats. Data are presented as the mean \pm standard deviation $(\mathrm{n}=3)$. ${ }^{* *} \mathrm{P}<0.01$ vs. sham group, ${ }^{\# \#} \mathrm{P}<0.01$ vs. model group. W/D, wet/dry; ALI, acute lung injury.

A

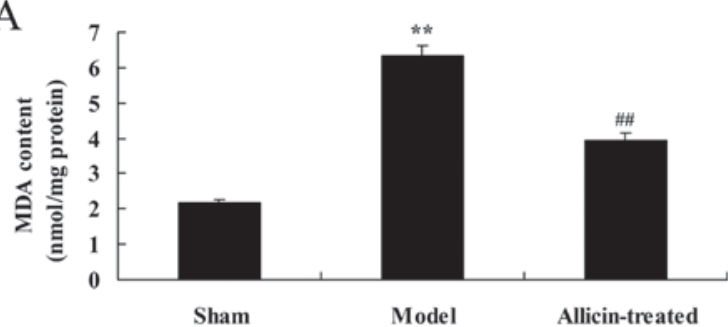

C

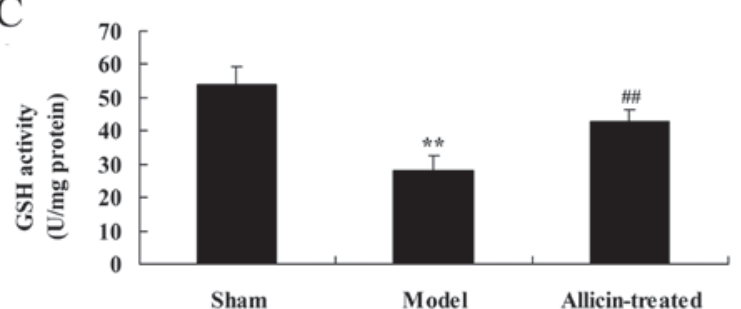

B

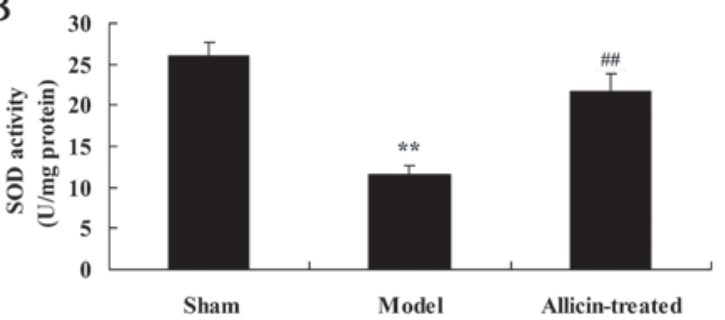

$\mathrm{D}$

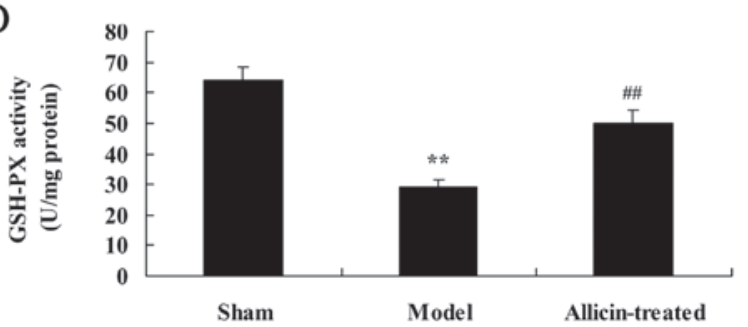

Figure 5. Effect of allicin on the levels of MDA and the activity of SOD, GSH and GSH-PX in the BALF of ALI model neonatal rats. (A) Effect of allicin on the levels of MDA in the BALF of ALI model neonatal rats. Effect of allicin on the activity of (B) SOD, (C) GSH and (D) GSH-PX in the BALF of ALI model neonatal rats. Data are presented as the mean \pm standard deviation $(\mathrm{n}=3){ }^{*}{ }^{* *} \mathrm{P}<0.01$ vs. sham group, ${ }^{\# \#} \mathrm{P}<0.01$ vs. model group. MDA, malondialdehyde; SOD, superoxide dismutase; GSH, glutathione; GSH-PX, glutathione peroxidase; BALF, bronchoalveolar lavage fluid; ALI, acute lung injury.

decrease at $24 \mathrm{~h}$ after treatment with allicin, compared with the sham group (Fig. 2). Allicin inhibited the mortality rate of LPS-treated rats at various time-points, compared with the ALI model group (Fig. 2).

Effect of allicin on histological examination of LPS-treated neonatal rats. As demonstrated in Fig. 3, alveolar edema, hemorrhage, wall thickening and hyperinflation, and inflammatory cell infiltration into the alveolar and interstitial spaces, of the model group were higher compared with the sham group. However, treatment with allicin reduced the LPS-induced alveolar edema, hemorrhage, wall thickening and hyperinflation, and inflammatory cell infiltration into the alveolar and interstitial spaces, in ALI rats (Fig. 3).

Effect of allicin on lung W/D ratio and protein concentration in LPS-induced ALI neonatal rats. As demonstrated in Fig. 4, the lung W/D ratio and lung protein concentration in the ALI model group were higher compared with 
A

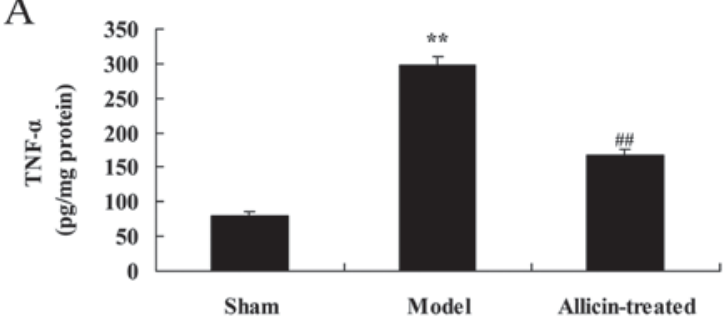

$\mathrm{C}$

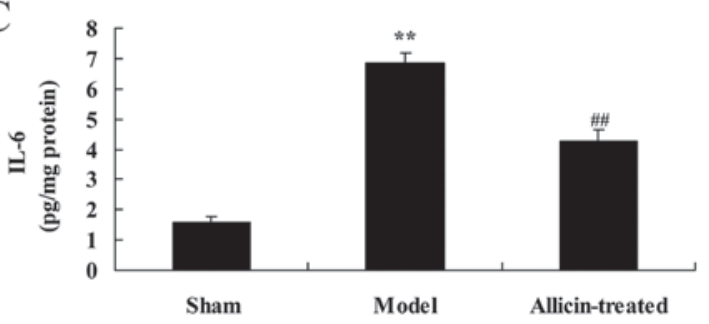

B

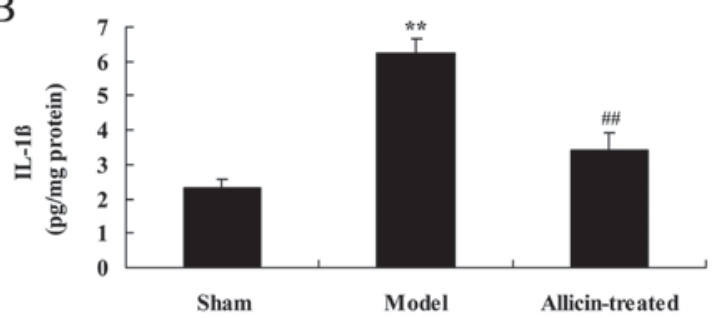

$\mathrm{D}$

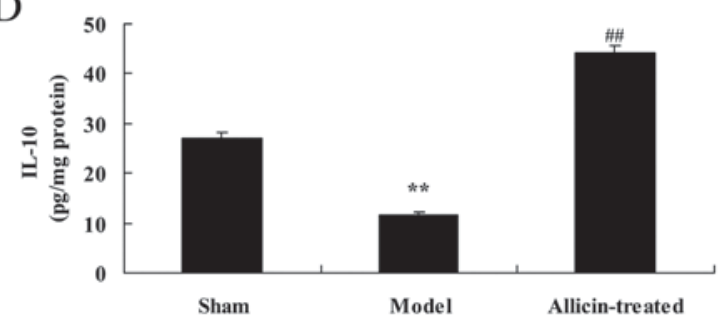

Figure 6. Effect of allicin on inflammation in the BALF of ALI model neonatal rats. Effect of allicin on (A) TNF- $\alpha$, (B) IL-1 $\beta$, (C) IL-6 and (D) IL-10 in the BALF of ALI model neonatal rats. Data are presented as the mean \pm standard deviation $(n=3) .{ }^{* *} \mathrm{P}<0.01$ vs. sham group, ${ }^{\# \#} \mathrm{P}<0.01 \mathrm{vs}$. model group. $\mathrm{BALF}$, bronchoalveolar lavage fluid; ALI, acute lung injury; TNF, tumor necrosis factor; IL, interleukin.

A

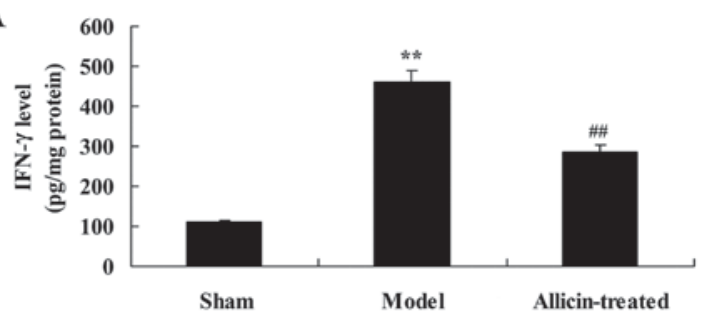

$\mathrm{C}$

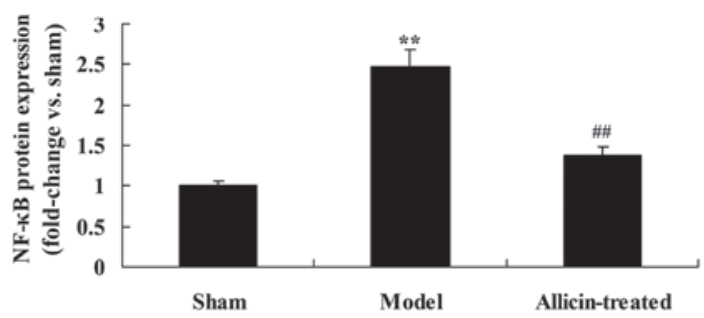

$\mathrm{B}$

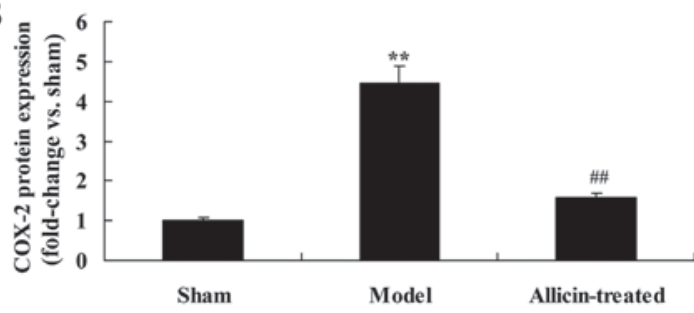

D

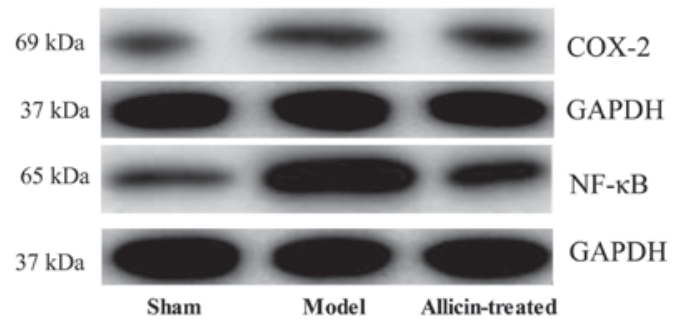

Figure 7. Effect of allicin on inflammation pathway components in the BALF of ALI model neonatal rats. (A) Effect of allicin on IFN- $\gamma$ levels in the BALF of ALI model neonatal rats. The protein expression of (B) COX-2 and (C) NF- $\mathrm{kB}$ in the BALF of ALI model neonatal rats was quantified by western blotting and densitometric analysis. (D) Representative western blot bands for COX-2 and NF- $\mathrm{kB}$ protein expression in the BALF of ALI model neonatal rats. Data are presented as the mean \pm standard deviation $(\mathrm{n}=3)$. ${ }^{* *} \mathrm{P}<0.01$ vs. sham group, ${ }^{\# /} \mathrm{P}<0.01$ vs. model group. BALF, bronchoalveolar lavage fluid; ALI, acute lung

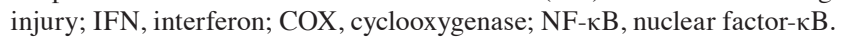

the sham group (Fig. 4). However, allicin administration significantly decreased the elevations in lung W/D ratio and lung protein concentration, compared with the ALI model group (Fig. 4).

Effect of allicin on the levels of MDA, and activity of SOD, glutathione $(G S H)$ and GSH peroxidase (GSH-PX), in the BALF of LPS-treated neonatal rats. Compared with the sham group, the levels of MDA were significantly increased, and SOD, GSH and GSH-PX activity was significantly inhibited, in the ALI model group (Fig. 5). However, allicin treatment significantly reversed the effects of LPS on MDA levels, and SOD, GSH and GSH-PX activity, compared with the ALI model group (Fig. 5).
Effect of allicin on inflammation in the BALF of LPS-treated neonatal rats. As demonstrated in Fig. 6A-C, the levels of TNF- $\alpha$, IL- $1 \beta$ and IL- 6 in the BALF of ALI model rats were significantly increased, compared with the sham group. However, TNF- $\alpha$, IL-1 $\beta$ and IL-6 levels were significantly suppressed by treatment with allicin, compared with the ALI model group (Fig. 6A-C). In addition, the levels of IL-10 in the BALF of ALI model rats were lower compared with the sham group (Fig. 6D). Treatment with allicin significantly increased IL-10 levels in ALI rats, compared with the ALI model group (Fig. 6D).

Effect of allicin on inflammatory pathways. The levels of interferon (IFN)- $\gamma$, and the protein expression of cyclooxygenase 
A

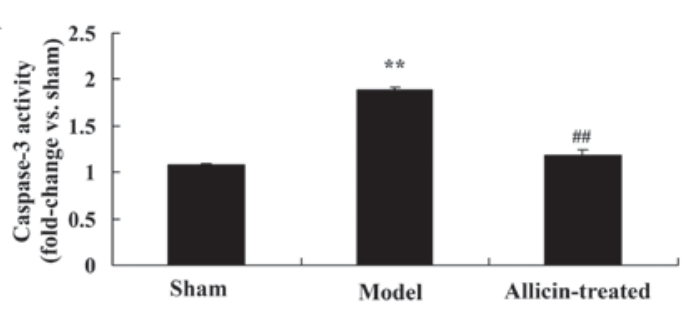

$\mathrm{C}$

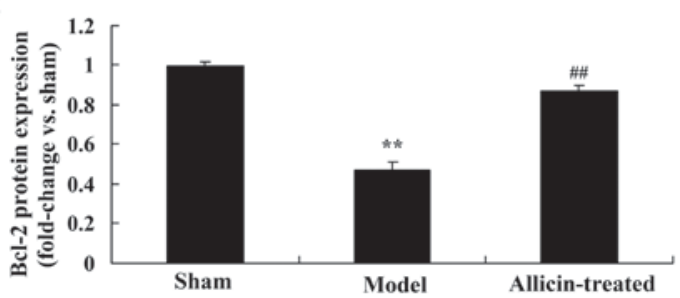

B

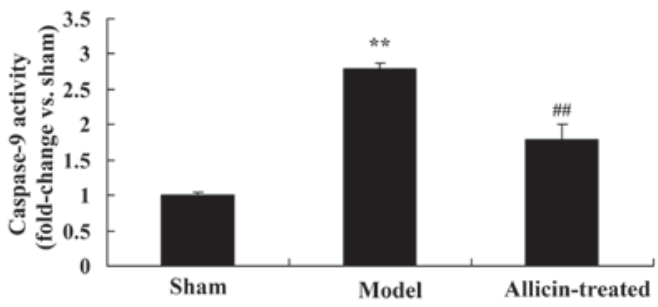

$\mathrm{D}$

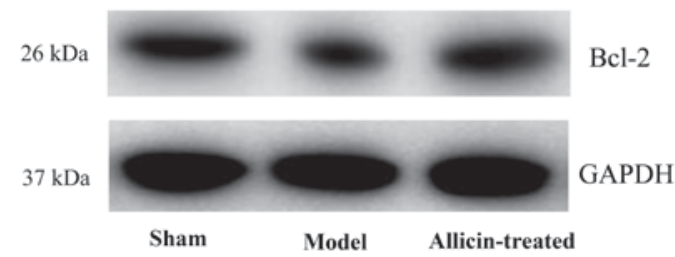

Figure 8. Effect of allicin on the activity of caspase-3/-9 and protein expression of Bcl-2 in the BALF of ALI model neonatal rats. Effect of allicin on (A) caspase-3 and (B) caspase-9 activity in the BALF of ALI model neonatal rats, as determined by ELISA. (C) Bcl-2 protein expression in the BALF of ALI model neonatal rats was quantified by western blotting and densitometric analysis. (D) Representative western blot bands for Bcl-2 protein expression in the BALF of ALI model neonatal rats. Data are presented as the mean \pm standard deviation $(n=3){ }^{* *} \mathrm{P}<0.01$ vs. sham group, ${ }^{\# \#} \mathrm{P}<0.01$ vs. model group. BALF, bronchoalveolar lavage fluid; ALI, acute lung injury.

A

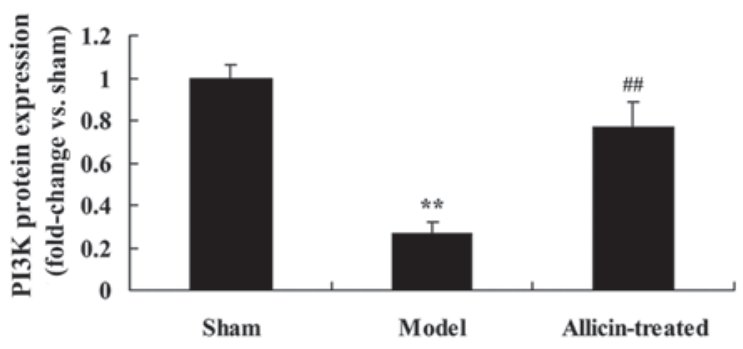

$\mathrm{C}$

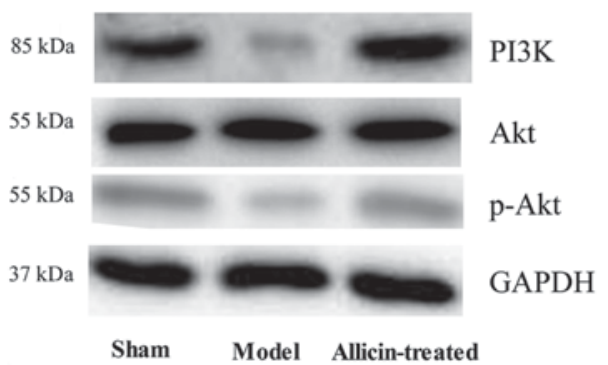

$\mathrm{B}$

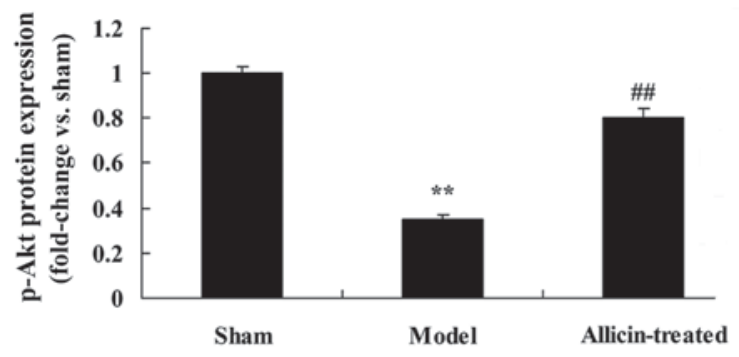

Figure 9. Effect of allicin on the protein expression of PI3K and p-Akt in the BALF of ALI model neonatal rats. The effect of allicin on (A) PI3K and (B) p-Akt protein expression in the BALF of ALI model neonatal rats was quantified by western blotting and densitometric analysis. (C) Representative western blot bands for the protein expression of PI3K and p-Akt in the BALF of ALI model neonatal rats. Data are presented as the mean \pm standard deviation ( $\mathrm{n}=3$ ). ${ }^{* * *} \mathrm{P}<0.01$ vs. sham group; ${ }^{\# \#} \mathrm{P}<0.01$ vs. model group.

(COX)-2 and nuclear factor- $\kappa \mathrm{B}(\mathrm{NF}-\kappa \mathrm{B})$, in the BALF were significantly promoted in ALI model rats, compared with the sham group (Fig. 7). However, allicin treatment significantly reduced IFN- $\gamma$ levels, and COX-2 and NF- $\kappa B$ protein expression, in ALI rats, compared with the ALI model group (Fig. 7).

Effect of allicin on anti-apoptotic protein of Bcl-2 and caspase-3/-9 pathway. The results of ELISA and western blotting also demonstrated that upregulation of caspase-3/-9 activity and the inhibition of $\mathrm{Bcl}-2$ protein expression, respectively, in the ALI model group compared with the sham group (Fig. 8). However, upregulation of caspase-3/-9 activity and the inhibition of $\mathrm{Bcl}-2$ protein expression were induced by allicin administration, compared with the ALI model group (Fig. 8).

Effect of allicin on the phosphatidylinositol 3-kinase (PI3K) and phosphorylated ( $p)$-Akt pathway. The protein expression of PI3K and p-Akt was significantly reduced in the ALI model group, compared with the sham group (Fig. 9). However, $\mathrm{PI} 3 \mathrm{~K}$ and p-Akt protein expression levels were significantly increased by treatment with allicin, compared with the ALI model group (Fig. 9). 


\section{Discussion}

Oxidative stress is an important mechanism in the pathogenesis of ALI (16). A large number of oxygen free radicals (OFR) are produced during the ALI process by various cell types within the vessel wall, including endothelial cells, vascular smooth muscle cells and cells of the outer membrane (17). The present study demonstrated that allicin reduced increases in the lung W/D ratio that were observed in the ALI model group, and identified that it may have potential as a drug for the treatment of ALI.

Oxidative stress leads to increases in cell permeability, cell edema and potentially lytic necrosis (18). The targets of OFR include nucleic acids, proteins and membrane lipids, and lipid peroxidation is one of the primary mechanisms of free radical damage (19). MDA is employed as a marker for lipid peroxidation to reflect the degree of oxidative stress injury following ALI. To combat oxidative stress injury, various endogenous cytoprotective substances are generated in the lung tissue, such as SOD, which may be used as an indicator of antioxidative activity (20). A dynamic equilibrium exists between oxidation and antioxidant systems in vivo; when these systems become imbalanced, tissue damage results and the lung is one of the most vulnerable target organs (21). A previous study demonstrated that MDA content was increased significantly in ALI rat lung homogenates, while SOD level was decreased significantly, which indicates that an imbalance between the oxidation and antioxidant system may be involved in the pathogenesis of ALI (22). Furthermore, the results of the current study demonstrated that allicin treatment significantly reversed the effects of ALI model induction on MDA, SOD, GSH and GSH-PX levels in ALI neonatal rats.

The development of ALI may be initiated by alveolar inflammation under the perinatal asphyxia or infection, as well as additional internal and external factors (23). The number and activation of neutrophils is increased, which increases the release of various inflammatory mediators, and epithelial and endothelial cells functions are subsequently damaged, which is a major mechanism in the pathogenesis of ALI (23). It is reported that IL-6 combines with heparin sulfate glycoprotein on the vascular endothelial cell surface, which has a potent chemotactic effect and causes endothelial cells to adhere to and activate neutrophils (24). Therefore, the release of IL-6 is considered to be an important factor in the pathogenesis of ALI (25). In addition, TNF- $\alpha$ also has an important role in the inflammatory lesion of tissue and promotes the release of other inflammatory mediators (26). The results of the present study demonstrated that allicin significantly suppressed TNF- $\alpha$, IL-1 $\beta$, IL- 6 and IFN- $\gamma$ levels, and COX-2 and NF- $\mathrm{KB}$ protein expression, and increased IL-10 levels, in ALI neonatal rats.

Apoptosis is a type of programmed cell death that is important in the development and injury of multicellular organisms in various tissues and organs (27). Caspases and the Bcl-2 protein family are closely associated with apoptosis (28). Apoptosis-associated genes are divided into apoptosis-promoting genes and apoptosis-inhibiting genes (29). Caspase-3 activation leads to the induction of cell apoptosis, and has an important role in the initiation and implementation of early apoptosis. Caspase-3 activation is a biochemical indicator of early apoptosis. Bcl-xl is an anti-apoptotic gene that exerts anti-apoptosis effects by inhibiting the activation of caspase proteases (30). Proapoptotic genes are dominant during early apoptosis, during which $\mathrm{Bcl}-\mathrm{xl}$ expression is decreased, and as apoptosis develops, the internal anti-apoptotic mechanism is activated (31). The present study demonstrated that allicin significantly increased the expression of the Bcl-2 anti-apoptotic protein and inhibited caspase-3/-9 activity in ALI model neonatal rats, which may occur via the PI3K/Akt pathway. Ding et al reported that allicin inhibited oxidative stress-induced mitochondrial dysfunction and apoptosis of osteoblast cells through PI3K/Akt signaling (32). The present study only analyzed the effects of allicin on PI3K/Akt signaling in ALI, which is a limitation of the study as allicin may also regulate additional signaling pathways to exert effects on inflammation, oxidative stress and apoptosis, which should be investigated in future studies.

In conclusion, the present study demonstrated that allicin treatment suppressed lung W/D ratio and the lung concentration of proteins in ALI neonatal rats. These beneficial effects may be due to its ability to inhibit oxidative stress and inflammation, and to inhibit apoptosis, including Bcl-2 expression, caspase-3/-9 activityin ALI neonatal rats, which may occur via the PI3K/Akt pathway. Therefore, allicin may have potential as a novel drug for the treatment of neonatal ALI.

\section{Acknowledgements}

Not applicable.

\section{Funding}

No funding was received.

\section{Availability of data and materials}

The analyzed data sets generated during the study are available from the corresponding author on reasonable request.

\section{Authors' contributions}

XW designed the experiment. XW, CZ, CC, YG, XM and CK performed experiments. XW analyzed the data and wrote the manuscript.

\section{Ethics approval and consent to participate}

The present study was approved by the Institutional Animal Care and Use Committee of The Second Affiliated Hospital of Xuzhou Medical University (Xuzhou, China).

\section{Consent for publication}

Not applicable.

\section{Competing interests}

The authors declare that they have no competing interests. 


\section{References}

1. Reisinger MW, Moss M and Clark BJ; National Heart, Lung, and Blood Institute Acute Respiratory Distress Syndrome Network Investigators: Brief versus full alcohol use disorders identification test in national heart, lung, and blood institute acute respiratory distress syndrome network clinical trials. Crit Care Med 43: e382-e385, 2015.

2. Bernard GR: Potential of N-acetylcysteine as treatment for the adult respiratory distress syndrome. Eur Respir J Suppl 11: 496s-498s, 1990

3. Braunschweig CA, Sheean PM, Peterson SJ, Gomez Perez S, Freels S, Lateef O, Gurka D and Fantuzzi G: Intensive nutrition in acute lung injury: A clinical trial (INTACT). JPEN J Parenter Enteral Nutr 39: 13-20, 2015

4. Schwameis R, Eder S, Pietschmann H, Fischer B, Mascher H, Tzotzos S, Fischer H, Lucas R, Zeitlinger M and Hermann R: A FIM study to assess safety and exposure of inhaled single doses of AP301-A specific ENaC channel activator for the treatment of acute lung injury. J Clin Pharmacol 54: 341-350, 2014

5. Piva JP, Garcia PC and Fiori H: Mechanical ventilation in children with acute respiratory distress syndrome: A huge gap between what we know and our practice!*. Pediatr Crit Care Med 14: 732-733, 2013.

6. Mulder HD, Augustijn QJ, van Woensel JB, Bos AP, Juffermans NP and Wösten-van Asperen RM: Incidence, risk factors, and outcome of transfusion-related acute lung injury in critically ill children: A retrospective study. J Crit Care 30: 55-59, 2015.

7. Lorenz RT and Cysewski GR: Commercial potential for Haematococcus microalgae as a natural source of astaxanthin Trends Biotechnol 18: 160-167, 2000.

8. Fitzgerald JC, Topjian AA, McInnes AD, Mattei P, McCloskey JJ, Friess SH and Kilbaugh TJ: Bi-caval dual lumen venovenous extracorporeal membrane oxygenation and high-frequency percussive ventilatory support for postintubation tracheal injury and acute respiratory distress syndrome. J Pediatr Surg 46: e11-e15, 2011.

9. Wallock-Richards D, Doherty CJ, Doherty L, Clarke DJ, Place M, Govan JR and Campopiano DJ: Garlic revisited: Antimicrobial activity of allicin-containing garlic extracts against Burkholderia cepacia complex. PLoS One 9: e112726, 2014.

10. Huang S, Dai Y, Zhang Z, Hao W and Chen H: Docosahexaenoic acid intake ameliorates ketamine-induced impairment of spatial cognition and learning ability in ICR mice. Neurosci Lett 580: $125-129,2014$

11. Chen S, Tang Y, Qian Y, Chen R, Zhang L, Wo L and Chai H: Allicin prevents $\mathrm{H}_{2} \mathrm{O}_{2}$-induced apoptosis of HUVECs by inhibiting an oxidative stress pathway. BMC Complement Altern Med 14: 321, 2014

12. Chu YL, Ho CT, Chung JG, Raghu R, Lo YC and Sheen LY: Allicin induces anti-human liver cancer cells through the p53 gene modulating apoptosis and autophagy. J Agric Food Chem 61: 9839-9848, 2013.

13. Feldberg RS, Chang SC, Kotik AN, Nadler M, Neuwirth Z, Sundstrom DC and Thompson NH: In vitro mechanism of inhibition of bacterial cell growth by allicin. Antimicrob Agents Chemother 32: 1763-1768, 1988.

14. Khodavandi A, Harmal NS, Alizadeh F, Scully OJ, Sidik SM, Othman F, Sekawi Z, Ng KP and Chong PP: Comparison between allicin and fluconazole in Candida albicans biofilm inhibition and in suppression of $H W P 1$ gene expression. Phytomedicine 19: 56-63, 2011

15. Elkayam A, Peleg E, Grossman E, Shabtay Z and Sharabi Y: Effects of allicin on cardiovascular risk factors in spontaneously hypertensive rats. Isr Med Assoc J 15: 170-173, 2013.

16. Rice TW, Wheeler AP, Thompson BT, deBoisblanc BP Steingrub J and Rock P; NIH NHLBI Acute Respiratory Distress Syndrome Network of Investigators: Enteral omega-3 fatty acid, gamma-linolenic acid, and antioxidant supplementation in acute lung injury. JAMA 306: 1574-1581, 2011.
17. Huang TY, Tsai PS, Wang TY, Huang CL and Huang CJ: Hyperbaric oxygen attenuation of lipopolysaccharide-induced acute lung injury involves heme oxygenase-1. Acta Anaesthesiol Scand 49: 1293-1301, 2005

18. Wang C, Wang HY, Liu ZW, Fu Y and Zhao B: Effect of endogenous hydrogen sulfide on oxidative stress in oleic acid-induced acute lung injury in rats. Chin Med J 124: 3476-3480, 2011.

19. Yamaoka S, Kim HS, Ogihara T, Oue S, Takitani K, Yoshida Y and Tamai H: Severe Vitamin E deficiency exacerbates acute hyperoxic lung injury associated with increased oxidative stress and inflammation. Free Radic Res 42: 602-612, 2008.

20. Balyasnikova IV, Visintine DJ, Gunnerson HB, Paisansathan C, Baughman VL, Minshall RD and Danilov SM: Propofol attenuates lung endothelial injury induced by ischemia-reperfusion and oxidative stress. Anesth Analg 100: 929-936, 2005.

21. Lingappan K, Jiang W, Wang L, Wang G, Couroucli XI, Shivanna B, Welty SE, Barrios R, Khan MF, Nebert DW, et al: Mice deficient in the gene for cytochrome P450 (CYP)1A1 are more susceptible than wild-type to hyperoxic lung injury: Evidence for protective role of CYP1A1 against oxidative stress. Toxicol Sci 141: 68-77, 2014.

22. Sunil VR, Vayas KN, Massa CB, Gow AJ, Laskin JD and Laskin DL: Ozone-induced injury and oxidative stress in bronchiolar epithelium are associated with altered pulmonary mechanics. Toxicol Sci 133: 309-319, 2013.

23. Saini Y, Greenwood KK, Merrill C, Kim KY, Patial S, Parameswaran N, Harkema JR and LaPres JJ: Acute cobalt-induced lung injury and the role of hypoxia-inducible factor 1alpha in modulating inflammation. Toxicol Sci 116: 673-681, 2010.

24. Lo Re S, Dumoutier L, Couillin I, Van Vyve C, Yakoub Y, Uwambayinema F, Marien B, van den Brûle S, Van Snick J, Uyttenhove C, et al: IL-17A-producing gammadelta T and Th17 lymphocytes mediate lung inflammation but not fibrosis in experimental silicosis. J Immunol 184: 6367-6377, 2010.

25. Misumi T, Tanaka T, Mikawa K, Nishina K, Morikawa O and Obara H: Effects of sivelestat, a new elastase inhibitor, on IL-8 and MCP-1 production from stimulated human alveolar epithelial type II cells. J Anesth 20: 159-165, 2006.

26. Li J, Zhao L, He X, Zeng YJ and Dai SS: Sinomenine protects against lipopolysaccharide-induced acute lung injury in mice via adenosine $\mathrm{A}_{2 \mathrm{~A}}$ receptor signaling. PLoS One 8: e59257, 2013.

27. Liu W, Dong M, Bo L, Li C, Liu Q, Li Z and Jin F: Epigallocatechin-3-gallate suppresses alveolar epithelial cell apoptosis in seawater aspiration-induced acute lung injury via inhibiting STAT1-caspase-3/p21 associated pathway. Mol Med Rep 13: 829-836, 2016

28. Damarla M, Parniani AR, Johnston L, Maredia H, Serebreni L, Hamdan O, Sidhaye VK, Shimoda LA, Myers AC, Crow MT, et al: Mitogen-activated protein kinase-activated protein kinase 2 mediates apoptosis during lung vascular permeability by regulating movement of cleaved caspase 3. Am J Respir Cell Mol Biol 50: 932-941, 2014.

29. Halimah E, Diantini A, Destiani DP, Pradipta IS, Sastramihardja HS, Lestari K, Subarnas A, Abdulah R and Koyama H: Induction of caspase cascade pathway by kaempferol-3-rhamnoside in LNCaP prostate cancer cell lines. Biomed Rep 3: 115-117, 2015.

30. Guo Q, Jin J, Yuan JX, Zeifman A, Chen J, Shen B and Huang J: VEGF, Bcl-2 and Bad regulated by angiopoietin-1 in oleic acid induced acute lung injury. Biochem Biophys Res Commun 413: 630-636, 2011

31. Qiu X, Li H, Tang H, Jin Y, Li W, YuSun, PingFeng, Sun X and Xia Z: Hydrogen inhalation ameliorates lipopolysaccharide-induced acute lung injury in mice. Int Immunopharmacol 11: 2130-2137, 2011.

32. Mangiacapra F, Colaiori I, Ricottini E, Balducci F, Creta A, Demartini C, Minotti G and Di Sciascio G: Heart Rate reduction by IVabradine for improvement of ENDothELial function in patients with coronary artery disease: The RIVENDEL study. Clin Res Cardiol 106: 69-75, 2017. 